\title{
Robust Optic Disk Segmentation from Colour Retinal Images
}

\author{
Gopal Datt Joshi \\ CVIT, IIIT Hyderabad \\ Hyderabad, India \\ gopal@research.iiit.ac.in
}

\author{
Rohit Gautam \\ CVIT, IIIT Hyderabad \\ Hyderabad, India \\ rohit_g@students.iiit.ac.in \\ S. R. Krishnadas \\ Aravind Eye Hospitals \\ Madurai, India \\ krishnadas@aravind.org
}

\author{
Jayanthi Sivaswamy \\ CVIT, IIIT Hyderabad \\ Hyderabad, India \\ jsivaswamy@iiit.ac.in
}

\begin{abstract}
We present a novel segmentation method to better capture the boundary of a non-homogeneous object such as the optic disk(OD), defined locally by two similar characteristic regions. Existing active contour models which utilise gradient information [12] or global region intensity [2] fail to localise aforementioned boundaries. We propose a region-based active contour model that uses local image information around each point of interest in multi-dimensional feature space. This model uses a local energy functional and level-set representation to achieve desired OD segmentation. The local energy functional defined on each image point provides sufficient information to determine a desired OD segmentation which is robust to the variations found in and around the OD region. This method has been evaluated against the segmentation provided by three medical experts on 138 retinal images. Both region and boundary-based assessment performed against two well established active contour models show strengths of the proposed method.
\end{abstract}

\section{INTRODUCTION}

The optic disk (OD) is an important structure in the human retina. It is the exit point of retinal nerve fibers from the eye, and the entrance and exit point for retinal blood vessels. Any change in the shape, depth or colour in or within OD is used by ophthalmologists to assess various retinal pathologies. Several attempts for automatic retinal image analysis and assessment have been made towards assisting ophthalmologists in several ways such as by reducing workload, by providing quantitative evaluation, etc,. One of the important steps in retinal image analysis is the OD segmentation. Other than being an indicator for various ophthalmic

* Corresponding author

Permission to make digital or hard copies of all or part of this work for personal or classroom use is granted without fee provided that copies are not made or distributed for profit or commercial advantage and that copies bear this notice and the full citation on the first page. To copy otherwise, to republish, to post on servers or to redistribute to lists, requires prior specific permission and/or a fee.

ICVGIP'10, December 12-15, 2010, Chennai, India

Copyright 2010 ACM 978-1-4503-0060-5/10/12 ...\$10.00.
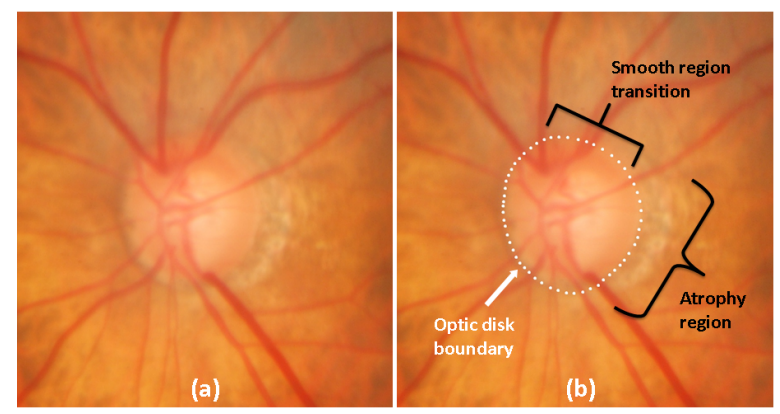

Figure 1: a) Original color retinal image b) Highlighting ill-defined boundary region and image variation near OD boundary due to atrophy (a pathological change).

pathologies, it acts as a reference to measure distances and identify other anatomical parts in retinal images (e.g. the fovea), for blood vessel tracking, etc.

A colour fundus (retinal) image (CFI) is a projection of retinal structures on 2-D color plane where the OD appears as a bright circular or elliptic region partially occluded by blood vessels as shown in Fig.1(a). OD segmentation is a challenging task mainly due to blood vessel occlusions, illdefined boundaries, image variations near disk boundaries due to pathological changes and variable imaging conditions. Specifically, occurrence of similar characteristics regions (Atrophy) near disk boundary, irregular disk shape and boundary are the most essential aspects to be addressed by a OD segmentation method. A sample image is shown in Fig 1 to illustrate the above conditions.

Lalonde et al. [6] propose an OD localisation scheme using Hausdorff based template matching and pyramidal decomposition. This method assumes a circular model to approximate OD region for which radius parameter was estimated from the localised region. Chrastek et al. [4] also consider a circular model and present a method consists of four steps: localization of the optic disc, nonlinear filtering, Canny edge detector and Hough transform. A similar method is proposed in [1] with an improved morphologicalbased pre-processing step.

A method proposed in [16] uses an intensity-based template matching to coarsely localise the OD boundary and 
then smoothen by an ellipse fitting step. Recently, few active contour based methods have been presented to better capture irregular OD shapes. Mendels et al.[12] use a greylevel mathematical morphology and extract the OD boundary using gradient vector flow (GVF) snake similar to the approach presented in [8]. A set of manually marked boundary points are used to initialise the snake. The accuracy of this method is highly dependent on the intialisation together with the sensitivity of the snake to the local energy minima which primarily arise due to gradient variations in and around the OD region. Osareh et al. [15] present improvements by automatically initialising boundary points using a template matching scheme. Lowell et al.[11] use an elliptical shape based deformable model to eliminate sensitivity to the local minima. A variational level-set based approach followed by a ellipse fitting step is presented in [21]. Li et al. [9][10] modify an active shape model (ASM) for the OD and obtain the optimal model parameters by fitting to the image data. Novo et al. [14] showed application of topological active net (TAN) model for the localisation and segmentation of the OD, together with the genetic algorithm scheme for its optimisation. Few energy terms are incorporated in the TAN model to impose circularity in expected segmentation. Enforcement of certain shape models helps the method to handle gradient variations within and around OD but altogether limits the extraction range of irregular OD shapes which occur commonly in a clinical scenario.

Juan et al.[22] propose a model-free snake approach and report improvements over their earlier active shape model (ASM) [9][10]. In this method, after each deformation, contour points are classified in a supervised manner into edgepoint cluster or uncertain-point cluster. The updating is only carried out on the contour points which belong to the edge-point cluster. Deformation of each point uses both global and local information to overcome local gradient variations. The successful results on both normal and challenging OD examples have been reported on which their earlier approach [9][10] was failing. This method shows promise is capturing a range of shape and image variations, however the accuracy in the segmentation is sensitive to the contour initialization.

To address various challenges associated with the object segmentation, various active models have been successfully employed for different vision applications. More recently, work in active contours has been focused on region-based approaches[2] inspired by the basic idea of the Mumford-Shah model [13]. In such models, foreground and background regions are modeled statistically and an energy functional is minimised to best separate foreground and background regions. Figure 2(a) shows a successful segmentation on an image with large gradient distractions near boundaries. The advantages in using the region-based approaches over edgebased methods include the following: a) feasibility of segmentation of color and multi-spectral images even in the absence of gradient-defined boundaries, b) lower sensitivity to contour initialisation and noise, and c) better ability to capture concavities of objects.

However, in cases where the object to be segmented cannot be easily distinguished in terms of global statistics, regionbased active contours may lead to erroneous segmentations. Figure 2(b) shows a failure example due to smooth region transition between object and background region. Yandong et. al [19] proposed a Chan and Vese (C-V) model [2] based

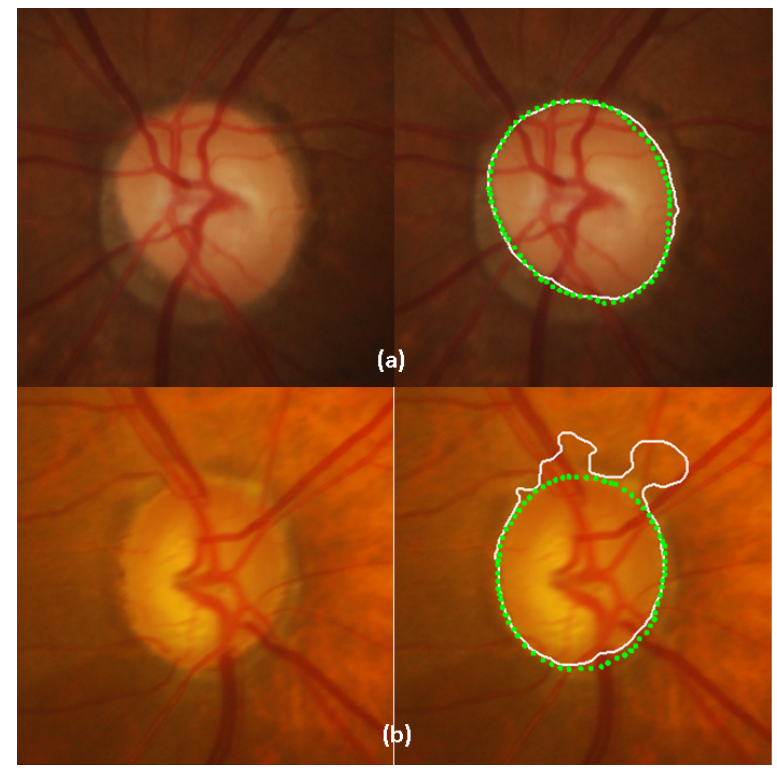

Figure 2: Sample results of $\mathrm{C}-\mathrm{V}$ active contour[2]. Green: Ground truth by an expert; White: Obtained result. a) First row: successful example, b) Second row: failure example.

OD segmentation method but imposed a circularity constraint on the active contour model to handle such situations.

In this paper, we propose a novel OD segmentation method based on $\mathrm{C}-\mathrm{V}$ model to improve the segmentation on the range of OD instances. The scope of $\mathrm{C}-\mathrm{V}$ model is extended by including image information at the support domain around each point of interest. The model is further refined to differentiate the OD region from the similar characteristic regions around it by integrating information from the multiple image feature channels. This method does not impose any shape constraint to the underlying model hence makes a good choice for OD segmentation. In the next section, we give brief detail about the original $\mathrm{C}-\mathrm{V}$ model.

\section{BACKGROUND}

Consider a vector-valued image $I: \Omega \rightarrow \mathbb{R}^{d}$ where $\Omega \subset$ $\mathbb{R}^{n}$ in the image domain, and $d \geq 1$ is the dimension of the vector $I(\mathrm{x})$. Let $C(s):[0,1] \rightarrow \mathbb{R}^{2}$ be a piecewise parameterized $C^{1}$ curve. For a gray valued image, the $\mathrm{C}-\mathrm{V}$ model [2] defines an energy functional as:

$$
\begin{array}{r}
E\left(c^{+}, c^{-}, C\right)=\lambda^{+} \int_{\text {inside }(C)}\left|I(\mathrm{x})-c^{+}\right|^{2} d \mathrm{x} \\
+\lambda^{-} \int_{\text {outside }(C)}\left|I(\mathrm{x})-c^{-}\right|^{2} d \mathrm{x} \\
+\mu \text { length }(C)
\end{array}
$$

where inside $(C)$ and outside $(C)$ represent the region inside and outside of the contour $\mathrm{C}$, respectively and $c^{-}$and $c^{+}$are two constants that approximate the image intensity inside and outside of the contour.

This model assumes that an image consists of statistically homogeneous regions and therefore lacks the ability to deal with objects having intensity inhomogeneity. Figure 2(b) 
shows an example. Intensity inhomogeneous is very common is natural images especially in OD region its the most occurring phenomena. Recently there have been some attempts to improve C-V model for such situations [7] [20] [17]. Here, the basic idea is to use local instead of global image intensity into the region-based active contour model. These methods report significant improvement in the segmentation over original $\mathrm{C}-\mathrm{V}$ model for segmenting objects with heterogeneous intensity statistics. However other than intensity heterogeneity within $\mathrm{OD}$, smooth region transition at boundary locations and occurrence of similar characteristic regions near the OD boundaries (atrophy) make OD segmentation a much more difficult case altogether. Figure $1 \& 4$ illustrate OD examples with additional challenges. The local intensity based statistics [7] [20] is not sufficient the discriminate atrophy regions.

Here, we present a region-based active contour model which uses local image information at the support domain around each point of interest (POI) inspired by localised C-V models [7] [20]. We propose to use a richer form of local image information gathered over multi-dimensional feature space. The intention is to represent the POI more holistically by including descriptions of the intensity, colour, texture, etc. This approach should yield a better discriminating representation of image regions and make the proposed model robust to the distractions found near the OD boundaries.

\section{LOCALISED AND VECTOR-VALUED C- V ACTIVE CONTOUR MODEL}

In this section, we present a region-based active contour model which constrains the local behavior of each point of interest $\mathrm{x}$ based on the image information at $\mathrm{x}$ and at its neighboring points. Let $\mathrm{x}$ and $\mathrm{y}$ denote two points in an image $I$. We define a local function $\kappa$ for each $\mathrm{x}$ as:

$$
\kappa(x, y)= \begin{cases}1 & \text { if }\|\mathrm{x}-y\| \leq r \\ 0 & \text { otherwise }\end{cases}
$$

where, $\kappa$ which defines the local image domain around a point $\mathrm{x}$ within a radius of $r$. This function will be 1 when the point $\mathrm{y}$ is within a radius of $\mathrm{r}$ centered at point $\mathrm{x}$ and 0 otherwise. Using the above function, the energy (mentioned in equ.(1)) for a point $\mathrm{x}$ is redefined as:

$$
\begin{aligned}
E_{\mathrm{x}}\left(h^{+}, h^{-}, C\right)= & \lambda^{+} \int_{\Omega_{y}} \kappa(\mathrm{x}, y)\left|I(y)-h^{+}\right|^{2} d y \\
& +\lambda^{-} \int_{\Omega_{y}} \kappa(\mathrm{x}, y)\left|I(y)-h^{-}\right|^{2} d y
\end{aligned}
$$

where, $h^{-}$and $h^{+}$are two constants that approximate region intensities inside and outside of the contour $\mathrm{C}$ respectively, near the point $\mathrm{x}$. The local function ensures the values of $h$ that minimise $E_{\mathrm{x}}\left(h^{+}, h^{-}, C\right)$ is only influenced by the image information within the local domain. This way the behavior of any individual point is constrained by the regional information from a local domain. This helps in capturing local boundaries which get missed by a $\mathrm{C}-\mathrm{V}$ model due to small difference in the global statistics of interior and exterior region of the contour.

Now, we incorporate information from a multi-dimensional feature space, in the above model. Let $I_{i}$ be the $i^{\text {th }}$ feature of an image on $\Omega$ with $\mathrm{i}=1, \ldots, d$. The extension of the above model to the vector case is:

$$
\begin{aligned}
\bar{E}_{\mathrm{x}}\left(h^{+}, h^{-}, C\right)= & \frac{1}{d} \sum_{i=1}^{d} \lambda_{i}^{+} \int_{\Omega_{y}} \kappa(\mathrm{x}, y)\left|I_{i}(y)-h_{i}^{+}\right|^{2} d y \\
& +\frac{1}{d} \sum_{i=1}^{d} \lambda_{i}^{-} \int_{\Omega_{y}} \kappa(\mathrm{x}, y)\left|I_{i}(y)-h_{i}^{-}\right|^{2} d y
\end{aligned}
$$

where $h^{+}=\left(h_{1}^{+}, \ldots, h_{d}^{+}\right)$and $h^{-}=\left(h_{1}^{-}, \ldots, h_{d}^{-}\right)$are two constant vectors approximating region feature values inside and outside of the contour $\mathrm{C}$ respectively in each feature space. The $\lambda_{i}^{+}>0$ and $\lambda_{i}^{+}>0$ are weight parameters for the error term defined for each feature space.

The above energy $\bar{E}_{\mathrm{x}}$ defined for a point $\mathrm{x} \in \Omega$ can be minimised when this point is exactly on the object boundary and values of $h^{+}$and $h^{-}$are optimally chosen. The integral of $\bar{E}_{\mathrm{x}}$ over all points x is minimised to obtained entire object boundary. This is defined as:

$$
E\left(h^{+}, h^{-}, C\right)=\int_{\Omega} \bar{E}_{\mathrm{x}}\left(h^{+}, h^{-}, C\right) d(\mathrm{x})
$$

This energy is converted to an equivalent level-set formulation [3] for curve evolution.

\subsection{Level-set formulation of the model}

In level-set formulation, a contour $C \subset \Omega$ is represented by the zero level set of Lipschitz function $\phi: \Omega \rightarrow \mathbb{R}$. In this representation, the energy functional $\bar{E}_{\mathrm{x}}\left(h^{+}, h^{-}, C\right)$ in (3) can be rewritten as

$$
\begin{array}{r}
\bar{E}_{\mathrm{x}}\left(h^{+}, h^{-}, \phi\right)=\frac{1}{d} \sum_{i=1}^{d} \lambda_{i}^{+} \int_{\Omega_{y}} \kappa(\mathrm{x}, y)\left|I_{i}(y)-h_{i}^{+}\right|^{2} H(\phi(y)) d y \\
+\frac{1}{d} \sum_{i=1}^{d} \lambda_{i}^{-} \int_{\Omega_{y}} \kappa(\mathrm{x}, y)\left|I_{i}(y)-h_{i}^{-}\right|^{2}(1-H(\phi(y))) d y
\end{array}
$$

where $\mathrm{H}$ is the heaviside function. Now, energy term in (4) can be written as:

$$
\begin{array}{r}
E\left(h^{+}, h^{-}, \phi\right)=\int_{\Omega} \bar{E}_{\mathrm{x}}\left(h^{+}, h^{-}, \phi\right) \\
=\int_{\Omega}\left[\frac{1}{d} \sum_{i=1}^{d} \lambda_{i}^{+} \int_{\Omega_{y}} \kappa(\mathrm{x}, y)\left|I_{i}(y)-h_{i}^{+}\right|^{2} H(\phi(y)) d y\right] d \mathrm{x} \\
+\int_{\Omega}\left[\frac{1}{d} \sum_{i=1}^{d} \lambda_{i}^{-} \int_{\Omega_{y}} \kappa(\mathrm{x}, y)\left|I_{i}(y)-h_{i}^{-}\right|^{2}(1-H(\phi(y))) d y\right] d \mathrm{x}
\end{array}
$$

A distance regularization term [20] is incorporated to penalise the deviation of $\phi$ from a signed distance function characterised by the following integral:

$$
\xi(\phi)=\int_{\Omega} \frac{1}{2}(|\nabla \phi(\mathrm{x})|-1)^{2} d \mathrm{x}
$$

To regularise the zero level contour of $\phi$, a length of zero level curve of $\phi$ is also added which is given as:

$$
\zeta(\phi)=\int_{\Omega} \delta \phi(\mathrm{x})|\nabla \phi(\mathrm{x})| d \mathrm{x}
$$

Now, we define the entire energy functional

$$
F\left(h^{+}, h^{-}, \phi\right)=E\left(h^{+}, h^{-}, \phi\right)+\alpha \xi(\phi)+\beta \zeta(\phi) ;
$$


where $\alpha$ and $\beta$ are non-negative constants. This energy functional is minimised to the optic disk boundary. The minimisation method and performed approximations are provided in the appendix.

\section{OPTIC DISK LOCALISATION AND CON- TOUR INTIALISATION}

The first step is to localise OD region and define region of interest on which further processing shall be carried out. The red colour plane of CFI gives good definition of OD region thus a good choice for the OD localisation task. The contour initialisation is the next essential step to initiate active contour evolution. In our method, we perform localisation and initialisation steps together by performing circular Hough transform [5] on the gradient map.

The vessel points are identified and masked using standard vessel segmentation technique. The value at a vessel point is interpolated using near-by regions such that gradient values arise due to vessel structures can be eliminated prior to Hough transformation. Next, a canny edge detector at a very low threshold is applied on the pre-processed (vessel-free) image to get edge points. On these points, a circular Hough transform is applied for a range of expected OD radius $\left(r_{\min }\right.$ to $\left.r_{\max }\right)$. This range is chosen based on the retinal image resolution.

For each edge point, we draw a circle with center in the point with a radius $r$. This circle is drawn in the parameter space where $\mathrm{x}$ and $\mathrm{y}$ represent image axis and the $\mathrm{z}$ axis is the radii. At the coordinates which belong to the perimeter of the drawn circle we increment the value in the accumulator matrix. Once edge point and every desired radius is used, the accumulator will now contain numbers corresponding to the number of circles passing through the individual coordinates. Thus the higher numbers correspond to the center of the potential circles in the image. Since, edge points are mainly dominated by the OD region, we select OD center which has maximum value in the accumulator matrix. Next, the edges near the identified center location in the image domain are used to estimate the radius of the circle. The circle points are identified using estimated radius and further used to initialise active contour mentioned in section 3 .

\section{OPTIC DISK SEGMENTATION}

A multi-dimensional image representation is obtained from different colour and texture feature space. In normal image conditions, red colour plane gives a better contrast of OD region. To better characterise OD in pathological situations, two different texture representations are derived.

First, Gaussian filter responses obtained at three finer scales $\sigma=\sqrt{2}, 2,2 \sqrt{2}$ and are integrated together. Second, we use a special class of texture filter bank proposed in [18] defined as:

$$
L(r, \sigma, \tau)=L_{0}(\sigma, \tau)+\cos \left(\frac{\pi \tau r}{\sigma}\right) e^{-\left(\frac{r^{2}}{2 \sigma^{2}}\right)}
$$

where $\tau$ is the number of cycles of the harmonic function within the Gaussian envelope of the filter, commonly used in the context of Gabor filters. $L_{0}(\sigma, \tau)$ is added to obtain a zero DC component. These filter responses are obtained at three pairs $(\sigma, \tau)=(4,2),(6,3),(8,3)$ and are integrated together to capture finer regularity in the texture profile. These responses are computed on the red colour plane of

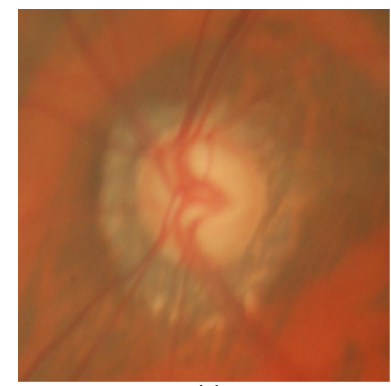

(a)

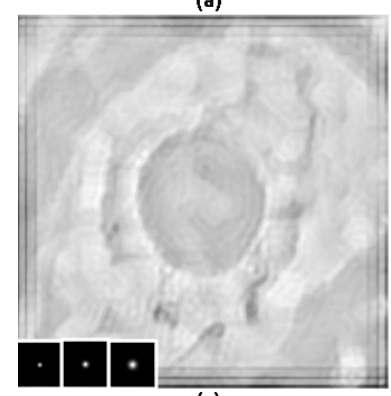

(c)

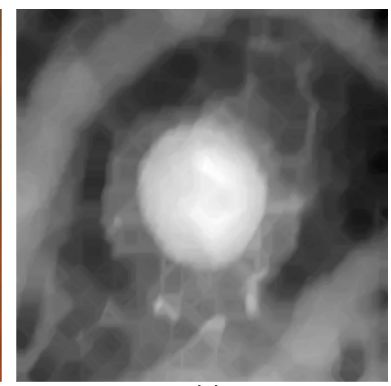

(b)

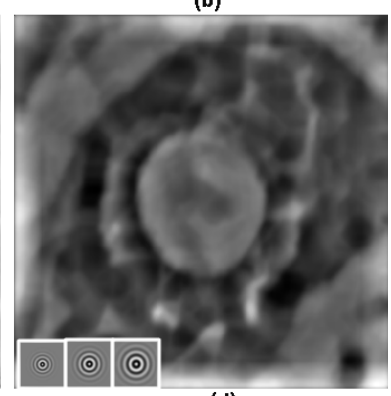

(d)
Figure 3: Different feature space representation for the OD region. a) Original colour image, b) Red colour plane, c) Texture space-1, and d) Texture space-2.

the image. Prior to this computation, the points belong to the vessel region are removed and interpolated as mentioned in section 4. In general, choice of texture representation is driven by the capability it provide to distinguish OD region from the various atrophy regions occurring near to the OD. Figure 3 shows three different feature space representations.

Now, a image point $\mathrm{x}$ is represented by a three element vector where value of individual vector element is taken from red colour plane texture feature space $1 \& 2$, respectively. This vector-valued image is used by the active contour model presented in section 3 to get the OD boundary.

\section{EXPERIMENTS AND RESULTS}

\subsection{Datasets}

We evaluate method's performance on a dataset collected from a local eye hospital. It consists of a total of $138 \mathrm{im}-$ ages of size $2896 \times 1944$ and are mainly OD centric. The markings of OD boundary have been taken from three eye experts with varying clinical experience. To compensate inter-observer marking variations, we derive an average OD boundary for each image by averaging boundaries obtained by three experts, called as gold standard. The evaluation has been carried out against three experts individually and also against gold standard. Different comparisons have been made with two known active contour models: a) gradient vector flow (GVF) [12], and b) C-V model [2]. To only assess the strength of individual active contour model, curve initialisation and performed pre-processing are kept same for each model.

\subsection{Experiments}

The radius defined for the function $\kappa$ is kept 40 pixels for all the reported experiments. Figure 4 shows few sample results obtained by three different active contour models. 


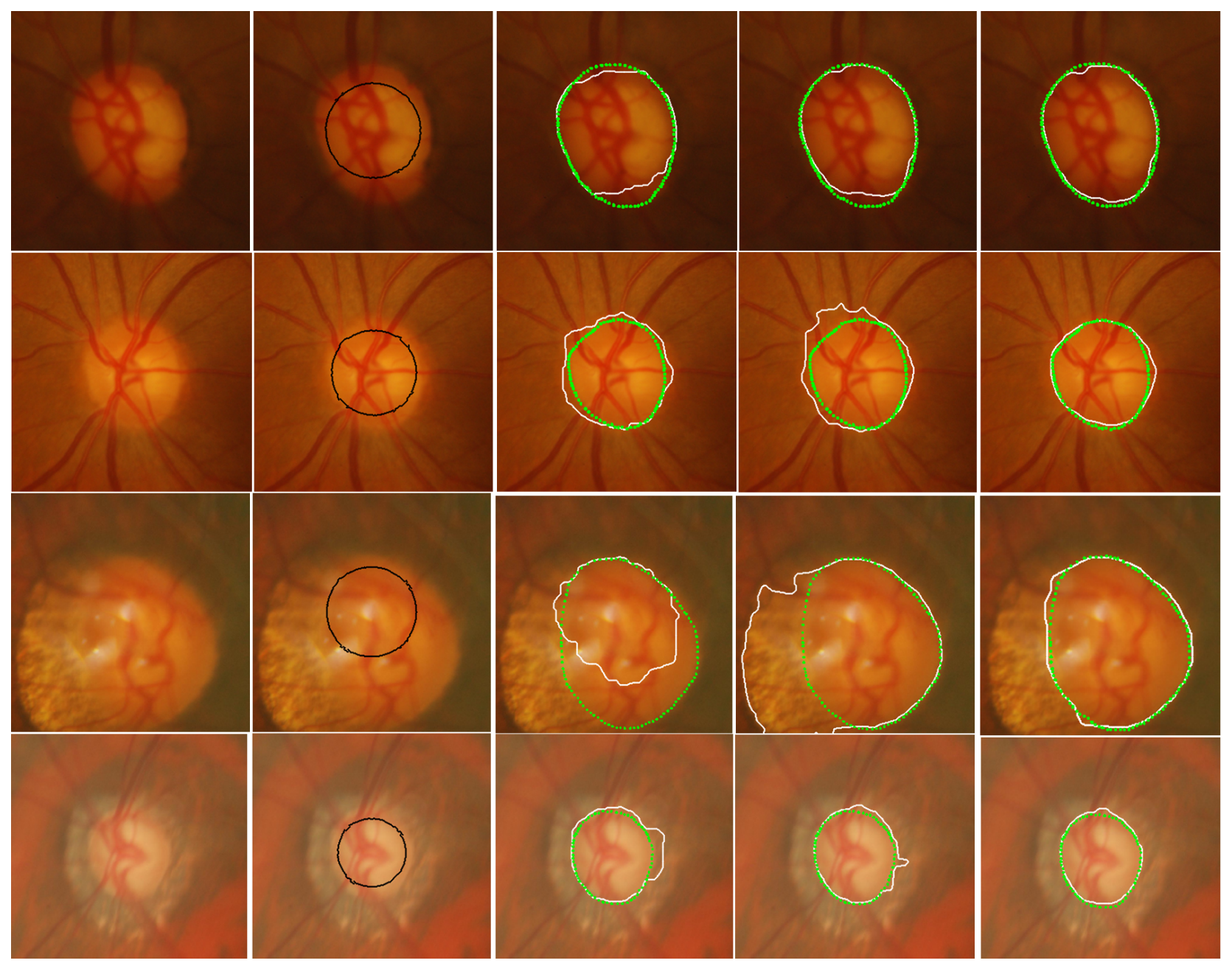

Figure 4: First column: original image; Second column: initialised contour; Third column: GVF results; Fourth column: C-V model results; Fifth column: proposed method results. Green colour indicates boundary marked by an expert and white colour indicates obtained boundary by a method. The last two rows show the high atrophy cases.

Second column illustrates initialised contour obtained by the scheme mentioned in section 4.

First row presents an example of irregular shape OD with a high gradient variations near the initialised contour. The GVF model fails to capture entire OD region due to local gradient minima. $\mathrm{C}-\mathrm{V}$ model is able to handle local gradient variations however low bright regions get excluded due to a subtle difference present between average intensity of the detected foreground and background regions. The proposed method better captures boundary except at the boundary regions which are occluded by thick blood vessels. This situation mainly arise due to the pre-processing carried out to suppress the vessel pixels. The vessel pixels at the boundary are usually get interpolated by the background pixels (which are outside the disk boundary) therefore considered background by the proposed method. Second row presents an example of fuzzy OD boundary where proposed method optimally capture the OD boundary compared to other methods. Third and fourth row show two successful segmentation results on two challenging atrophy cases.

Figure 5 shows an example to demonstrate inter-observer variability (subjectivity) present in the experts' marking.
This example has a good definition of OD boundary and it is carefully selected to demonstrate subjectivity, a well known aspect in medical image analysis. This subjectivity mainly arise due to expert's level of familiarity with the marking tool and their clinical experience. We compute a average OD boundary called as gold standard from three experts' boundaries to compensate subjectivity aspect. The obtained result by our method shows better boundary consensus with the gold standard compared to individual expert markings.

\subsubsection{Quantitative Evaluation}

A quantitative analysis is performed on total 138 images to assess overall performance of the method. This evaluation is carried out in two ways: a) region and b) boundary-based.

In region-based evaluation, we compute pixel-wise precision and recall values which are defined as:

$$
\text { Precision }=\frac{t p}{t p+f p} \quad \text { Recall }=\frac{t p}{t p+f n}
$$

where $t p=$ no. of true positive, $f p=$ no. of false positive and $\mathrm{fn}=$ no. of false negative pixels. Table 1 shows average precision and recall values obtained by three methods. A 


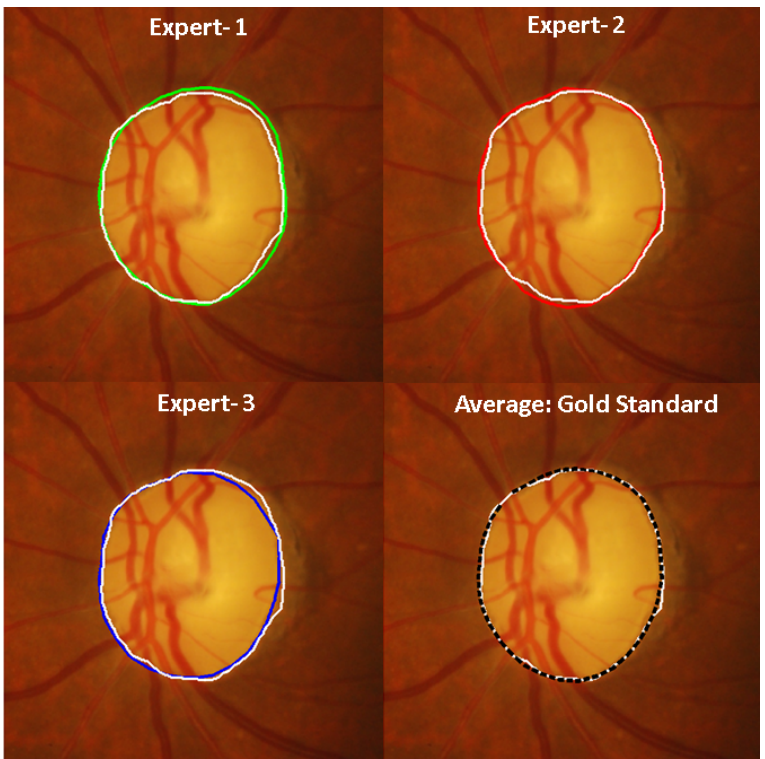

Figure 5: Evaluation against three experts and a gold standard.

Table 1: Average precision and recall computed over 138 images.

\begin{tabular}{|l|c|c|c|c|}
\hline & Expert-1 & Expert-2 & Expert-3 & $\begin{array}{c}\text { Gold } \\
\text { Standard }\end{array}$ \\
\hline GVF & $0.99 / 0.82$ & $0.99 / 0.80$ & $0.99 / 0.84$ & $0.99 / 0.83$ \\
C-V model & $0.96 / 0.94$ & $0.97 / 0.92$ & $0.95 / 0.96$ & $0.96 / 0.95$ \\
Ours & $0.98 / 0.94$ & $0.99 / 0.92$ & $0.97 / 0.96$ & $0.98 / 0.96$ \\
\hline
\end{tabular}

high precision and low recall value obtained by GVF model indicates a under-segmentation case which is mainly due to the local gradient minimas usually present within the OD region. Overall, our method achieves higher precision and recall values against four ground truths. To better appreciate results, we compute a single performance measure called traditional F-score (F) that is the harmonic mean of precision and recall. It is defined as:

$$
F=2 \frac{\text { Precision } \cdot \text { Recall }}{\text { Precision }+ \text { Recall }}
$$

This is also known as the $F_{1}$ score, because recall and precision are evenly weighted. Table 2 shows that proposed method overall gives better performance against two other models. Since dataset contains $95.4 \%$ of the images with normal condition, the difference in the obtained scores are not so prominent. However on challenging images, our method shows significant improvement in the segmentation results (can be seen from the Fig. 4).

We measure distance between two closed boundary curves to evaluate the accuracy of boundary localisation. Let $C_{g}$

Table 2: F-score computed over 138 images.

\begin{tabular}{|l|c|c|c|c|}
\hline & Expert-1 & Expert-2 & Expert-3 & $\begin{array}{c}\text { Gold } \\
\text { Standard }\end{array}$ \\
\hline GVF & 0.90 & 0.88 & 0.91 & 0.90 \\
C-V model & 0.95 & 0.94 & 0.96 & 0.96 \\
Ours & 0.96 & 0.95 & 0.97 & 0.97 \\
\hline
\end{tabular}

Table 3: Average boundary distance computed in radial direction.

\begin{tabular}{|l|c|c|c|c|}
\hline & Expert-1 & Expert-2 & Expert-3 & $\begin{array}{c}\text { Gold } \\
\text { Standard }\end{array}$ \\
\hline GVF & 0.332 & 0.353 & 0.301 & 0.312 \\
C-V model & 0.148 & 0.156 & 0.135 & 0.131 \\
Ours & 0.129 & 0.142 & 0.108 & 0.111 \\
\hline
\end{tabular}

be the boundary curve marked by the expert and $C_{o}$ be the curve obtained by a method. The distance (D) between two curves is defined as (in pixels):

$$
D=\frac{1}{L} \sum_{\theta=1}^{\theta_{n}} \sqrt{\left(d_{g}^{\theta}\right)^{2}+\left(d_{o}^{\theta}\right)^{2}}
$$

where, $d_{g}^{\theta}$ and $d_{o}^{\theta}$ are the distance from centroid of curve $C_{g}$ to points on $C_{g}$ and $C_{o}$, respectively in the direction of $\theta$. $\mathrm{L}$ is a non-zero constant used for value normalisation. The average distances computed against 4 ground truth boundary curves are given in Table 3 . It can be seen that the our method achieves better boundary localisation with the minimum distances to three experts and gold standard.

Both region and boundary-based evaluation show that the presented model achieves better segmentation compared to two existing active contour models.

\section{CONCLUSIONS}

In this work, we presented a novel, active contour model to achieve robust OD segmentation. We have extended the scope of $\mathrm{C}-\mathrm{V}$ model by including image information at the support domain around each point of interest. This model has been further strengthened by the integration of information from the multiple image feature channels.

The presented method captures OD boundary in a unified manner for both normal and challenging cases without imposing any shape constraint on the segmentation result, unlike the earlier methods. The method has been tested on a dataset of size 138 images and assessed against OD boundary marked by three medical experts. The comparison results show that the proposed method is more robust and accurate than other two models overall, and particularly in the cases of atrophy. This establishes its strengths for OD segmentation. Since the proposed modification of the C-V model is general, its scope is not limited to OD boundary detection but is widely applicable to other segmentation applications, especially in the medical imaging domain.

\section{APPENDIX}

The Heaviside function $\mathrm{H}$ in Eq. (5) is approximated by a smooth function $H_{\varepsilon}$ defined by

$$
H_{\varepsilon}(\mathrm{x})=\frac{1}{2}\left[1+\frac{2}{\pi} \arctan \left(\frac{\mathrm{x}}{\varepsilon}\right)\right]
$$

The derivative of $H_{\varepsilon}$ is the following smooth funtion

$$
\delta_{\varepsilon}(\mathrm{x})=H_{\varepsilon}^{\iota}(\mathrm{x})=\frac{1}{\pi} \frac{\varepsilon}{\varepsilon^{2}+\mathrm{x}^{2}}
$$

The approximation of $H, \delta$ by $H_{\varepsilon}, \delta_{\varepsilon}$ respectively, in Eq. (5) and Eq. (8) gives an approximated form of energy functional given in Eq. (9). 


$$
F_{\varepsilon}\left(h^{+}, h^{-}, \phi\right)=E_{\varepsilon}\left(h^{+}, h^{-}, \phi\right)+\alpha \xi(\phi)+\beta \zeta_{\varepsilon}(\phi) ;
$$

The value for $\varepsilon$ is chosen 1 for a good approximation [20]. This energy functional is minimised to find the OD boundary.

GRADIENT DESCENT FLOW: The gradient descent method is used to minimise the approximated energy functional. For a fixed level set function $\phi$, functional Eq.(12) is minimised w.r.t the functions $h_{i}^{+}$and $h_{i}^{-}$for $i=1,2, \ldots, d$. We obtain

$$
\begin{gathered}
h_{i}^{+}=\frac{\kappa(\mathrm{x}, y) *\left[H_{\varepsilon}(\phi(y)) I_{i}(y)\right]}{\kappa(\mathrm{x}, y) *\left[H_{\varepsilon}(\phi(y))\right]} \\
h_{i}^{-}=\frac{\kappa(\mathrm{x}, y) *\left[\left(1-H_{\varepsilon}(\phi(y))\right) I_{i}(y)\right]}{\kappa(\mathrm{x}, y) *\left[1-H_{\varepsilon}(\phi(y))\right]}
\end{gathered}
$$

Keeping $h_{i}^{+}$and $h_{i}^{-}$fixed and minimising the energy functional Eq.(12) w.r.t to $\phi$, the obtained gradient vector flow is:

$$
\begin{array}{r}
\frac{\partial \phi}{\partial t}=-\delta_{\varepsilon}(\phi)\left(e^{+}-e^{-}\right)+\alpha \delta_{\varepsilon}(\phi) \operatorname{div}\left(\frac{\nabla \phi}{|\nabla \phi|}\right) \\
+\beta\left(\nabla^{2} \phi-\operatorname{div}\left(\frac{\nabla \phi}{|\nabla \phi|}\right)\right)
\end{array}
$$

where $\delta_{\varepsilon}$ is the smooth Dirac function given in Eq.(11) and $e^{+}$and $e^{-}$are the functions below:

$$
e^{+}(\mathrm{x})=\frac{1}{d} \sum_{i=1}^{d} \lambda_{i}^{+} \int_{\Omega_{y}} \kappa(\mathrm{x}, y)\left|I_{i}(y)-h_{i}^{+}\right|^{2} d y
$$

and

$$
e^{-}(\mathrm{x})=\frac{1}{d} \sum_{i=1}^{d} \lambda_{i}^{-} \int_{\Omega_{y}} \kappa(\mathrm{x}, y)\left|I_{i}(y)-h_{i}^{-}\right|^{2} d y
$$

where $h_{i}^{+}$and $h_{i}^{-}$are given by Eq.(13) and Eq.(14), respectively.

\section{REFERENCES}

[1] R. A. Abdel-Ghafar and T. Morris. Progress towards automated detection and characterization of the optic disc in glaucoma and diabetic retinopathy. Informatics for Health and Social Care, 32(1):19-25, 2007.

[2] T. Chan and L. Vese. Active contours without edges. IEEE Trans. Image Processing, 10(2):266-277, 2001.

[3] T. F. Chan, B. Y. Sandberg, and L. A. Vese. Active contours without edges for vector-valued images. Journal of Visual Communication and Image Representation, 11(2):130-141, 2000.

[4] R. Chrt'astek, M. Wolf, K. Donath, G. Michelson, and H. Niemann. Optic disc segmentation in retinal images. Proc. BVM, pages 263-266, 2002.

[5] R. O. Duda and P. E. Hart. Use of the hough transformation to detect lines and curves in pictures. Communications of the ACM, 15(1):11-15, 1972.

[6] M. Lalonde, M. Beaulieu, and L. Gagnon. Fast and robust optic disc detection using pyramidal decomposition and hausdorff-based template matching. IEEE Trans Med Imaging. 20(11):1193-1200, 2001.
[7] S. Lankton and A. Tannenbaum. Localizing region-based active contours. IEEE Trans Image Process., 17(11):2029-2039, 2008.

[8] S. Lee and M. Brady. Optic disk boundary detection. Proc. BMVC, pages 359-362, 1991.

[9] H. Li and O. Chutatape. Boundary detection of optic disk by a modified ASM method. Pattern Recognition, 36(9):2093-2104, 2003.

[10] H. Li and O. Chutatape. A model-based approach for automated feature extraction in fundus images. IEEE Trans. Biomedical Engineering, 51(2):246-254, 2004.

[11] J. Lowell, A. Hunter, D. Steel, A. Basu, R. Ryder, E. Fletcher, and L. Kennedy. Optic nerve head segmentation. IEEE Trans. Medical Imaging, 23(2):256-264, 2004.

[12] F. Mendels, C. Heneghan, and J. P. Thiran. Identification of the optic disc boundary in retinal images using active contours. Proc. IMVIP, pages 103-115, 1999.

[13] D. Mumford and J. Shah. Optimal approximation by piecewise smooth functions and associated variational problems. Commun. Pure Appl. Math, 42:577-685, 1989.

[14] J. Novo, M. Penedo, and J. Santos. Localisation of the optic disc by means of GA-optimised topological active nets. Image and Vision Computing, 27(10):1572-1584, 2009.

[15] A. Osareh, M. Mirmehdi, B. Thomas, and R. Markham. Colour morphology and snakes for optic disc localisation. Proc. MIUA, pages 21-24, 2002.

[16] P. Pallawala, W. Hsu, M. Lee, and K. Eong. Automated optic disc localization and contour detection using ellipse fitting and wavelet transform. Proc. ECCV, pages 139-151, 2004.

[17] C. Sagiv, N. A. Sochen, and Y. Y. Zeevi. Integrated active contours for texture segmentation. IEEE Transactions on Image Processing, 15(6):1633-1646, 2006.

[18] C. Schmid. Constructing models for content-based image retrieval. Proc. CVPR, 2:39-45, 2001.

[19] Y. Tang, X. Li, A. von Freyberg, and G. Goch. Automatic segmentation of the papilla in a fundus image based on the $\mathrm{C}-\mathrm{V}$ model and a shape restraint. Proc. ICPR, pages 183-186, 2006.

[20] L. Wang, L. He, A. Mishra, and C. Li. Active contours driven by local gaussian distribution fitting energy. Signal Processing, 89(12):2435-2447, 2009.

[21] D. Wong, J. Liu, J. Lim, X. Jia, F. Yin, H. Li, and T. Wong. Level-set based automatic cup-to-disc ratio determination using retinal fundus images in argali. Proc. EMBC, pages 2266-2269, 2008.

[22] J. Xu, O. Chutatape, E. Sung, C. Zheng, and P. Chew. Optic disk feature extraction via modified deformable model technique for glaucoma analysis. Pattern Recognition, 40(7):2063-2076, 2007. 